\title{
Robust finite-time guidance against maneuverable targets with unpredictable evasive strategies
}

DOI:

10.1016/j.ast.2018.04.004

\section{Document Version}

Accepted author manuscript

Link to publication record in Manchester Research Explorer

\section{Citation for published version (APA):}

Zhang, R., Wang, J., Li, H., Li, Z., \& Ding, Z. (2018). Robust finite-time guidance against maneuverable targets with unpredictable evasive strategies. Aerospace Science and Technology.

https://doi.org/10.1016/j.ast.2018.04.004

\section{Published in:}

Aerospace Science and Technology

\section{Citing this paper}

Please note that where the full-text provided on Manchester Research Explorer is the Author Accepted Manuscript or Proof version this may differ from the final Published version. If citing, it is advised that you check and use the publisher's definitive version.

\section{General rights}

Copyright and moral rights for the publications made accessible in the Research Explorer are retained by the authors and/or other copyright owners and it is a condition of accessing publications that users recognise and abide by the legal requirements associated with these rights.

\section{Takedown policy}

If you believe that this document breaches copyright please refer to the University of Manchester's Takedown Procedures [http://man.ac.uk/04Y6Bo] or contact uml.scholarlycommunications@manchester.ac.uk providing relevant details, so we can investigate your claim.

\section{OPEN ACCESS}




\title{
Robust finite-time guidance against maneuverable targets with unpredictable evasive strategies
}

\author{
Ran Zhang ${ }^{\mathrm{a}}$, Jiawei Wang ${ }^{\mathrm{a}}$, Huifeng $\mathrm{Li}^{\mathrm{a}, *}$, Zhenhong $\mathrm{Li}^{\mathrm{b}}$, Zhengtao Ding ${ }^{\mathrm{b}}$ \\ ${ }^{a}$ School of Astronautics, Beihang University, Beijing 100191, P. R. China \\ ${ }^{b}$ School of Electrical and Electronic Engineering, University of Manchester, Manchester M13 9PL, UK
}

\begin{abstract}
This paper presents a robust finite-time guidance (RFTG) law to a short-range interception problem. The main challenge is that the evasive strategy of the target is unpredictable because it is determined not only by the states of both the interceptor and the target, but also by external un-modeled factors. By robustly stabilizing a line-of-sight rate, this paper proposes an integrated continuous finite-time disturbance observer/bounded continuous finite-time stabilizer strategy. The design of this integrated strategy has two points: 1) effect of a target maneuver is modeled as disturbance and then is estimated by the second-order homogeneous observer; 2) the finite-time stabilizer is actively coupled with the observer. Based on homogeneity technique, the local finite-time input-to-state stability is established for the closed-loop guidance system, thus implying the proposed RFTG law can quickly render the LOS rate within a bounded error throughout intercept. Moreover, convergence properties of the LOS rate in the presence of control saturation are discussed. Numerical comparison studies demonstrate the guidance performance.
\end{abstract}

Keywords: Robust finite-time guidance, Maneuverable target, Unpredictable evasive strategy, Finite-time input-to-state stability

\section{Introduction}

Interception of a maneuverable target is one of essential questions in the study of homing guidance. With the advancement of artificial intelligence, propulsion, composite material, etc, evasive capability of the target has been increasing at a rapid pace, and poses a challenging problem. This new guidance problem is referred to as Unpredictable Maneuverable Target Interception (UMTI) herein; that is, the interceptor cannot predict the evasive strategy of the target. The existing evasive strategies consist of conventional maneuver models $[1,2]$ and optimal evasive strategies $[3,4,5]$. The conventional maneuver models, such as a step maneuver, depend on prescribed maneuvers. The optimal evasive strategies are completely determined by the relative motion information of the interceptor and the target. These two types cannot be applied to the UMTI since both of them do not explicitly take account of the external un-modeled factors, particularly involving the deterministic yet unknown. In addition, considering that maneuvering capability of the target is comparable to that of the interceptor, the interceptor should be capable of exploiting its limit maneuverable capability, that is, control saturation.

\footnotetext{
* Corresponding author

Email addresses: zhangran@buaa.edu.cn (Ran Zhang), j.wang@buaa.edu.cn (Jiawei Wang), lihuifeng@buaa.edu.cn (Huifeng Li), zhenhong.li@postgrad.manchester.ac.uk

(Zhenhong Li), zhengtao.ding@manchester.ac.uk (Zhengtao Ding)
}

This paper is concerned with designing a robust guidance law to achieve the UMTI. In the literature, a number of guidance laws have been investigated to deal with the problems of the maneuverable target interception. These guidance laws can be roughly classified into two categories: relative-motion-prediction-based guidance law (RMP-GL) and manifold-stabilization-based guidance law (MS-GL). The RMP-GL usually obtains an optimal solution subject to a predetermined interception engagement (such as an ideal collision triangle scenario) using prediction of the relative motion between the interceptor and the target. The design tools of the RMP-GLs mainly include optimal control theory and differential game theory. For example, in [6], assuming an explicit model of the target maneuver and a first-order missile dynamics, the interception problem is formulated as a linear quadratic control problem; in [4], a nonlinear 3D-vector guidance law is designed, which is an optimal strategy pair in the sense of the saddle-point inequality. The RMP-GLs, however, cannot be applied to the UMTI since the prediction of the intercept motion (including a time-to-go and an intercept point) is difficult to carry out due to significant uncertainties of the target motion.

With respect to the MS-GLs, they render the interceptortarget relative motion around a prescribed manifold by compensating for (or suppressing) adverse effect of various disturbances and uncertainties related to the target maneuver. The design tools mainly consist of adaptive control, sliding mode control, high-gain control, etc. In [7], 
the authors parameterize upper bounds of the target acceleration and then develop the resulting parameter adaptive laws, thus handling the target maneuver. As such, the control saturation and the speed of the parameter adaptation are two fundamental concerns in adaptive control design $[8,9,10]$. To address these two concerns, in [11], a set of relative-state-dependent basis functions is selected to represent the target acceleration, and then a weight vector adaptive law is derived using optimal modification technique. Although the adaptive guidance laws work well in the above-mentioned scenarios, they have three drawbacks herein: 1) the guidance performance heavily depends on the convergence of adaptive parameters, while these parameters are easily prone to diverge when the jerk of the maneuverable target is considerable; 2) the robustness to the target maneuver relies on the parameterization of the target maneuver, while it is very hard to accurately parameterize the target maneuver under study; 3) the order of the closed-loop guidance system will inevitably increase as the dimension of the adaptive parameters grows.

The sliding mode control is used to suppress the effect of the target maneuver in $[12,13]$. In [14], the highorder sliding mode control is adopted to estimate and to compensate for the effect of the target maneuver. In this regard, the sliding mode guidance laws are upper-bounddependent. For the UMTI, the upper bounds of the target acceleration are required to be chosen relatively big for the sake of completely canceling the adverse effects of the target maneuver. Consequently, the resulting control is conservative and may induce severe chattering due to various kinds of modeling imperfections [15]. As another way, a smooth sliding mode guidance law is proposed in [16], in which an adaptive law is used to estimate the upper bound of the target maneuver. However, the adaptive parameter may diverge under the situation of control saturation.

In [17], the high-gain control is used to ensure input-tostate stability for a closed-loop LOS rate dynamics, and thereby the residual error of the LOS rate can be made sufficiently small in the presence of the target maneuver. In [18], a finite-time guidance is proposed to nullify the LOS rate, and the convergence boundary layer is theoretically analyzed. The guidance performance of the highgain control, however, may deteriorate due to measurement noise when the system bandwidth is excessively enlarged. In fact, it is difficult to make a reasonable trade-off between the disturbance suppression and the noise attenuation without the priori information of the evasive strategy of the target.

Following the technical route of the MS-GLs, a robust finite-time guidance (RFTG) law is presented in this paper. An integrated continuous finite-time disturbance observer (CFTDO)/bounded continuous finite-time stabilizer (BCFTS) strategy is proposed. The CFTDO is designed to estimate the disturbances that are the effects of the target maneuvers by making the observation-error dynamics behave as a second-order homogeneous system. The BCFTS uses a typical first-order homogeneous system to specify the finite-time stability of the nominal guidance system in consideration. Both the CFTDO and the BCFTS are based on non-smooth yet continuous feedback control. Such non-smooth feedback control has a favorable characteristic: its equivalent control gain increases as the feedback error decreases. In contrast to existing smooth feedback controls, the non-smooth feedback control may have better convergence and robustness $[19,20]$ and is suitable to be applied to the UMTI. Given these virtues, a few guidance laws based on the non-smooth feedback control have been designed. For example, the finite-time convergence of the LOS rate is realized in [12], but the effect of the target maneuver is suppressed using the conventional sliding mode control; in [21], using the high-gain control, the authors present a guidance law with finite-time input-to-state stability (FTISS). Note that the robustness of these two guidance laws is routinely guaranteed by the sliding mode control or the high-gain control. As discussed previously, these two control methods have their own drawbacks when adopted in the UMTI.

To address the preceding drawbacks, the integrated strategy is put forward with two key ideas: 1) the effect of the target maneuver is modeled as the disturbance and is estimated using the CFTDO; 2) the design of the BCFTS is coupled with that of the CFTDO. An unique characteristic of this integrated strategy is that it can explicitly deal with the interplay between the CFTDO and the BCFTS. This characteristic is different from the existing finite-time disturbance observer-based control methodologies (e.g., [22, 23, 24, 25, 26]), which design the observer and the finite-time controller in a decoupled fashion: they are based on a zero-observation-error assumption. However, the observation errors cannot be perfectly canceled in practice, especially concerning the UMTI. Nevertheless, the proposed RFTG law can avoid such a restricted assumption because of the integrated design adopted herein.

In addition, the proposed integrated strategy has several appreciable advantages in the current scenario. The BCFTS, which uses the control saturation to quickly stabilize the LOS rate, can take advantage of the limit of the maneuver capability of the interceptor. Regarding the CFTDO, it can rapidly estimate the disturbance of interest without suffering from the parameter convergence issues. Through cooperation between the BCFTS and the CFTDO, the resultant RFTG law is independent of both the explicit maneuver models and the upper bounds of the target maneuvers. Further, the RFTG law can guarantee locally FTISS in the presence of bounded derivative of the disturbance, implying the LOS rate can be rendered within a bounded error as quickly as possible. Numerical comparison results demonstrate the proposed RFTG law can guarantee the high-precision miss-distance.

This paper is organized as follows. We begin by formulating a new short-range interception problem. In particular, a notion of the unpredictable evasive strategies is introduced. The next section presents the robust finite-time guidance law. Then, the convergence analysis of the LOS 
rate is conducted in non-saturation and saturation cases. Finally, numerical comparison studies are performed to assess the guidance performance.

\section{Problem formulation}

The design and analysis of the RFTG law is based on the following assumptions:

Assumption 1. The motion of the interceptor and the target is in a planar plane.

Assumption 2. Both the interceptor and the target perform maneuvers orthogonal to their velocity vectors.

Assumption 3. The speeds of the interceptor and the target, $V_{M}$ and $V_{T}$, are constant, and $V_{M}>V_{T}$.

The equations of the relative motion between the interceptor and the target are given as follows [27]:

$$
\begin{aligned}
\dot{r} & =-V_{M} \cos \left(\lambda-\gamma_{M}\right)+V_{T} \cos \left(\lambda-\gamma_{T}\right), \\
\dot{\lambda} & =\frac{V_{M} \sin \left(\lambda-\gamma_{M}\right)}{r}-\frac{V_{T} \sin \left(\lambda-\gamma_{T}\right)}{r}, \\
\dot{\omega} & =\frac{-2 \dot{r} \omega}{r}-\frac{\cos \left(\lambda-\gamma_{M}\right) a_{M}}{r}+\frac{\cos \left(\lambda-\gamma_{T}\right) a_{T}}{r}, \\
\dot{\gamma}_{M} & =\frac{a_{M}}{V_{M}} \\
\dot{\gamma}_{T} & =\frac{a_{T}}{V_{T}},
\end{aligned}
$$

where $r$ is the interceptor-target range. $\lambda$ is the LOS angle. $\omega$ is the LOS rate: $\omega=\dot{\lambda}$. $\gamma_{M}$ and $\gamma_{T}$ are flight-path angles of the interceptor and the target. $a_{M}$ and $a_{T}$ are acceleration of the interceptor and the target, respectively. Herein, it is assumed that the variables, $r, \dot{r}, \lambda, \omega, V_{M}$, and $\gamma_{M}$, are available to the interceptor, while the states of the target, $V_{T}, \gamma_{T}$, and $a_{T}$ are unknown.

The target acceleration in the UMTI can be described by

$$
a_{T}=\Xi(x, \eta, \varpi, t),
$$

where $x$ represents the states of the interceptor and the target, such as $r, \lambda, V_{M}$, and $V_{T} . \eta$ represents the states of the prescribed maneuver models satisfying

$$
\dot{\eta}=f(\eta, \varsigma, t)
$$

where $\varsigma$ represents the parameter vector of the prescribed target maneuvers, such as frequency in a sinusoidal function. $\varpi$ represents the deterministic yet unknown maneuvers, such as an artificial-intelligence model and an eventtrigger-based model. It is $\varpi$ that makes the target acceleration unpredictable.

Assumption 4. The target acceleration $\Xi(x, \eta, \varpi, t)$ is continuously differentiable with respect to $t$, and is bounded, i.e., $|\Xi(x, \eta, \varpi, t)| \leq U_{T}$. The target jerk $\frac{\mathrm{d} \Xi(x, \eta, \varpi, t)}{\mathrm{d} t}$ is also bounded.
It is worth mentioning that the target maneuver model (6) almost encompasses existing target maneuver models used so far in the homing guidance literature. To be specific,

1. when $a_{T}=\Xi(x, t)$, the resulting maneuvers are determined by the information of the interceptor and the target. The optimal evasive strategies fall into this class;

2. when $a_{T}=\Xi(\eta, t)$, the resulting maneuvers represent the predictable maneuvers with the prescribed forms defined by (7), such as step maneuver, vertical$\mathrm{S}$ maneuver, spiraling maneuver, etc;

3. when $a_{T}=\Xi(x, \eta, \varpi, t)$, the resulting maneuvers are the model of the UMTI, which can not be predicted onboard due to unavailable mathematical description for $\varpi$.

Remark 1. From the view of the target maneuver models, the above three cases clarify the main differences between the UMTI and the existing interception problems. A specific target maneuver model concerning (6) is given in Section 5.1 to assess the guidance performance of the proposed guidance law.

At this point, we end this section by the objective of the present study: design a guidance law to intercept the target with acceptable small miss-distance in the presence of the target maneuvers described by (6).

\section{Robust finite-time guidance design}

In this section, the RFTG law is presented: first, the CFTDO is designed to estimate the effect of the target maneuvers; second, the integrated CFTDO/BCFTS strategy is proposed to robustly stabilize the LOS rate. Note that the design of both the CFTDO and the BCFTS is based on homogeneity technology, which is able to construct homogeneous dynamic systems with favorable finite-time stability. The fundamental notions used herein are briefly introduced in Appendix 7.1, and Appendix 7.2.

\subsection{Continuous finite-time disturbance observer (CFTDO)}

To estimate the effect of the target maneuver, the CFTDO is designed as follows:

$$
\begin{aligned}
\dot{\hat{\omega}}= & \frac{-2 \dot{r} \omega}{r}-\frac{\cos \left(\lambda-\gamma_{M}\right) a_{M}}{r}+\hat{d}_{T} \\
& +k_{1}|\omega-\hat{\omega}|^{\alpha} \operatorname{sgn}(\omega-\hat{\omega}), \\
\dot{\hat{d}}_{T}= & k_{2}|\omega-\hat{\omega}|^{2 \alpha-1} \operatorname{sgn}(\omega-\hat{\omega}),
\end{aligned}
$$

where $\hat{\omega}$ is the estimated LOS rate. $\hat{d}_{T}$ is the estimated effect of the target maneuver $d_{T}=\frac{\cos \left(\lambda-\gamma_{T}\right) a_{T}}{r} \cdot \frac{1}{2}<\alpha<$ 1. $k_{1}>0 . k_{2}>0 . \hat{\omega}(0)=\hat{\omega}_{0} \cdot \hat{d}_{T}(0)=\hat{d}_{T 0} \cdot \operatorname{sgn}(\cdot)$ is a signum function. 
Correspondingly, the observation-error dynamics are given by

$$
\begin{aligned}
& \dot{e}_{\omega}=e_{d}-k_{1}\left|e_{\omega}\right|^{\alpha} \operatorname{sgn}\left(e_{\omega}\right), \\
& \dot{e}_{d}=-k_{2}\left|e_{\omega}\right|^{2 \alpha-1} \operatorname{sgn}\left(e_{\omega}\right)+\delta(t),
\end{aligned}
$$

where $e_{\omega}=\omega-\hat{\omega} \cdot e_{d}=d_{T}-\hat{d}_{T} \cdot \delta(t)=\dot{d}_{T} \cdot e_{\omega}(0)=\omega(0)-$ $\hat{\omega}_{0}$. $e_{d}(0)=d_{T}(0)-d_{T 0}$. When $\delta(t)=0$, the obtained observation-error dynamics are reduced to a second-order homogeneous system whose origin is finite-time stable [20]. The stability analysis is carried out in Section 4.1. When $\delta(t) \neq 0$ and $|\delta(t)|$ is bounded, the observation errors can be made sufficiently small, which is proved in Section 4.2.

Remark 2. In light of the terms involving $\frac{1}{r}$ in (8), their value will be singular when $r=0$. Nevertheless, as discussed in the guidance literature, such as [28], this kind of singularity has little impact on the guidance performance. Besides, in practice, the engagement ends before $r=0$.

Remark 3. If $\alpha=1$, the observer (8) is a conventional Luenberger observer [29]. Compared with the Luenberger observer, the observer (8) has better performance in convergence and disturbance rejection by virtue of the nonsmooth feedback. In the case of $\alpha=\frac{1}{2}$, it is a secondorder sliding mode observer. Both the second-order sliding mode observer and the observer (8) can guarantee the finite-time convergence. Unlike the second-order sliding mode observer, the observer (8) does not require the upper bound of the target jerk, which is hard to be properly determined in the UMTI.

Remark 4. Another favorable property of the CFTDO is that it works well in the presence of the control saturation, which is observed in the numerical simulations.

\subsection{Integrated CFTDO/BCFTS strategy}

To stabilize the LOS rate with finite-time convergence, the robust finite-time guidance (RFTG) law is designed based on the integrated CFTDO/BCFTS strategy:

$$
\begin{aligned}
a_{M} & =U_{M} \operatorname{sat}\left(\frac{\phi\left(\lambda, \omega, \hat{d}_{T}\right)}{U_{M}}\right), \\
\phi\left(\lambda, \omega, \hat{d}_{T}\right) & =\frac{1}{\cos \left(\lambda-\gamma_{M}\right)}\left(-2 \dot{r} \omega+c_{1} r|\omega|^{\beta} \operatorname{sgn}(\omega)+r \hat{d}_{T}\right),
\end{aligned}
$$

where $U_{M}$ is the upper bound of the interceptor acceleration. $c_{1}>0.0<\beta<1$. $\operatorname{sat}(\cdot)$ is a saturation function: $\operatorname{sat}(y)=\min \{1,|y|\} \operatorname{sgn}(y)$.

As shown in (10), the RFTG law is composed by the CFTDO and the BCFTS. The task of the BCFTS is to stabilize the LOS rate within a small enough error in finite time. In particular, when $\left|\phi\left(\lambda, \omega, \hat{d}_{T}\right)\right|<U_{M}$, the resulting closed-loop dynamics of the LOS rate is

$$
\dot{\omega}=-c_{1}|\omega|^{\beta} \operatorname{sgn}(\omega)+e_{d} .
$$

If $e_{d}=0$, the resulting dynamics reduce to a typical firstorder homogeneous system with the finite-time stability, specifying the nominal performance of the BCFTS.

The following lemma states a particular homogeneous property of the integrated CFTDO/BCFTS strategy.

Lemma 1. Suppose that $\delta(t)=0$, and $\left|\phi\left(\lambda, \omega, \hat{d}_{T}\right)\right|<$ $U_{M}$. Then, by $\beta=\alpha$, the obtained closed-loop LOS rate dynamics are homogeneous of degree $1-\frac{1}{\alpha}$ with dilation $\left(\frac{1}{\alpha}, \frac{1}{\alpha}, 1\right)$.

Proof. Consider $\delta(t)=0$ and $\left|\phi\left(\lambda, \omega, \hat{d}_{T}\right)\right|<U_{M}$. Using the RFTG law (10), together with $\beta=\alpha$, the closed-loop closed-loop LOS rate dynamics are given by

$$
\begin{aligned}
\dot{\omega} & =-c_{1}|\omega|^{\alpha} \operatorname{sgn}(\omega)+e_{d}, \\
\dot{e}_{\omega} & =e_{d}-k_{1}\left|e_{\omega}\right|^{\alpha} \operatorname{sgn}\left(e_{\omega}\right), \\
\dot{e}_{d} & =-k_{2}\left|e_{\omega}\right|^{2 \alpha-1} \operatorname{sgn}\left(e_{\omega}\right) .
\end{aligned}
$$

By definition of the homogeneous vector field, it is straightforward to check that the dynamic system (12) is homogeneous of degree $1-\frac{1}{\alpha}$ with dilation $\left(\frac{1}{\alpha}, \frac{1}{\alpha}, 1\right)$.

If $\beta=\alpha$, this integrated CFTDO/BCFTS strategy has two advantages. First, it can explicitly cope with the interplay between the CFTDO and the BCFTS. Using the integrated strategy, the closed-loop system is constructed as a third-order homogeneous system with the finite-time stability. In the literature, the existing finite-time disturbance observer-based control methodologies (e.g., [23, 25, 26]) design the observer and the finite-time controller independently. In this case, the disturbances must be completely canceled by the observer in finite time, and then the finite-time controller is designed based on this zeroobservation-error assumption. However, such convenient design methodologies are not acceptable for the UMTI since the zero-observation-error assumption is not valid. Second, it can effectively reduce the so-called chattering effects in implementation. As pointed out by [15], the finite-time convergence is in conflict with the chattering suppression demand. A small power in the non-smooth feedback is the main reason. In this respect, increasing the power is a reasonable solution to relieving chattering. By virtue of the integrated design, the power can be increased without sacrificing the robustness, thereby reducing the chattering effects.

\subsection{Guidance scheme of RFTG law}

The RMP-GLs and the MS-GLs employ distinct guidance schemes. The scheme of the former is to first predict the relative motion between the interceptor and the target throughout the engagement, and then to correct the guidance command, so that the guided relative motion meets the prescribed guidance requirements. The MSGLs's guidance scheme is to directly maintain a desired relative motion relationship, which is a sufficient condition that can guarantee a successful interception. From 
the perspective of guidance performance, the RMP-GLs can achieve various optimal performance needs, while, except terminal constraints, the MS-GLs cannot attain other guidance performance needs. For example, the energy management of the RMP-GLs is superior to that of the MS-GLs. As a result, the trajectory curvature of the RMP-GLs would be significantly smaller than that of the MS-GLs, assuming that the knowledge of the target maneuver is available to the interceptor. If the future motion information of the target can be obtained onboard, the RMP-GLs will be the first choice.

The proposed RFTG law (10) follows the scheme of the MS-GLs due to the unpredictable target maneuvers in the UMTI. To achieve intercept, three conditions are needed: $\omega=0, \sin \left(\lambda-\gamma_{M}\right) a_{M} \geq \sin \left(\lambda-\gamma_{T}\right) a_{T}$, and $\dot{r}_{0}<0$, where $\dot{r}_{0}$ is the initial interceptor-target range rate. These three conditions can render the interceptortarget range rate negative, thus finally intercepting the target. The condition $\omega=0$ is an ideal relative motion relationship, and, in practical applications, it is suggested that the LOS rate $\omega$ should be stabilized with a sufficiently small error as quickly as possible. Furthermore, by examining the function $\phi\left(\lambda, \omega, \hat{d}_{T}\right)$, the non-smooth feedback control $c_{1} r|\omega|^{\alpha} \operatorname{sgn}(\omega)$ can lead to a large magnitude of the guidance command and likely cause the control saturation to happen at the start of the intercept. Nevertheless, the control saturation can effectively compensate for the effect of the target maneuver and stabilize the LOS rate using the limit of the interceptor's maneuverable capability. The rigorous convergence analysis of the LOS rate is performed in the following section.

\section{Convergence analysis of LOS rate}

This section presents the main theoretical results of the proposed RFTG law. Using homogeneity technique, global finite-time stability is established for the CFTDO. Based on Lyapunov theory, the convergence analysis of the LOS rate is conducted in the linear region of the saturation function in (10) and in the case of the control saturation, respectively.

\subsection{Global finite-time stability of CFTDO in the case of $\delta(t)=0$}

Suppose $\delta(t)=0$. Then, the observation-error dynamics are given by

$$
\begin{aligned}
& \dot{e}_{\omega}=e_{d}-k_{1}\left|e_{\omega}\right|^{\alpha} \operatorname{sgn}\left(e_{\omega}\right), \\
& \dot{e}_{d}=-k_{2}\left|e_{\omega}\right|^{2 \alpha-1} \operatorname{sgn}\left(e_{\omega}\right) .
\end{aligned}
$$

The CFTDO has the following property:

Theorem 1. The zero solution of the observation-error dynamics (13) is globally finite-time stable.

Proof. Consider a Lyapunov function candidate:

$$
V_{1}=\frac{k_{2}}{2 \alpha}\left|e_{\omega}\right|^{2 \alpha}+\frac{1}{2} e_{d}^{2}
$$

The derivative of $V_{1}$ along (13) is

$$
\dot{V}_{1}=-k_{1} k_{2}\left|e_{\omega}\right|^{3 \alpha-1} \text {. }
$$

Clearly, $\dot{V}_{1}$ is negative definite, and $\dot{V}_{1}=0$ means $e_{\omega}=0$. Using LaSalle's invariance theorem, the zero solution of the observation-error dynamics (13) is globally asymptotically stable. The degree of homogeneity of (13) is $1-\frac{1}{\alpha}<0$. Correspondingly, the finite-time stability of the zero solution can be established by the following lemma, which has been proved in [20] and is given below for the convenience of the reader.

Lemma 2. The zero solution of a homogeneous dynamic system is finite-time stable if and only if its zero solution is asymptotically stable and its degree of homogeneity is negative.

\subsection{Local finite-time input-to-state stability of RFTG law}

In this subsection, the convergence analysis is conducted in the linear region of the saturation function in (10). Accordingly, the guidance command (10) is replaced by

$$
a_{M}=\phi\left(\lambda, \omega, \hat{d}_{T}\right)
$$

Using the RFTG law (16), along with $\beta=\alpha$, the resulting closed-loop guidance system is given by

$$
\begin{aligned}
\dot{\omega} & =-c_{1}|\omega|^{\alpha} \operatorname{sgn}(\omega)+e_{d}, \\
\dot{e}_{\omega} & =e_{d}-k_{1}\left|e_{\omega}\right|^{\alpha} \operatorname{sgn}\left(e_{\omega}\right), \\
\dot{e}_{d} & =-k_{2}\left|e_{\omega}\right|^{2 \alpha-1} \operatorname{sgn}\left(e_{\omega}\right)+\delta(t) .
\end{aligned}
$$

Now, we are in a position to present the stability property of the RFTG law (16).

Theorem 2. The zero solution of the closed-loop guidance system (17) is locally finite-time input-to-state stable (FTISS).

Proof. By the Theorem 2 in [30], for system (13), there exists a homogeneous, and continuously differentiable Lyapunov function $V_{2}$ with dilation $\left(\frac{1}{\alpha}, 1\right)$, which satisfies

$$
\dot{V}_{2} \leq-\rho_{1} V_{2}^{\frac{2 \alpha}{\alpha+1}}
$$

where $\rho_{1}>0$, since system (13) is globally finite-time stable. (17):

Consider a Lyapunov function candidate for system

$$
V_{3}=\frac{1}{1+\alpha}|\omega|^{1+\alpha}+\Upsilon V_{2},
$$

where $\Upsilon>0$. Note that $V_{3}$ is positive definite and is homogeneous of $1+\frac{1}{\alpha}$ with dilation $\left(\frac{1}{\alpha}, \frac{1}{\alpha}, 1\right)$.

Next, we will show that, if $\Upsilon$ is sufficiently large, $V_{3}$ is a FTISS-Lyapunov function for (17). 
Let $x=\left(\omega, e_{\omega}, e_{d}\right)$. Using the Cauchy-Schwarz inequality [31], we can obtain

$$
\left|\frac{\mathrm{d} V_{3}}{\mathrm{~d} x}[0,0, \delta(t)]^{\top}\right| \leq \Upsilon U_{T}\left|\frac{\partial V_{2}}{\partial e_{d}}\right| .
$$

For a chosen compact set:

$$
\Sigma=\left\{\left(e_{\omega}, e_{d}\right) \in \mathbb{R}^{2}:\left|\frac{\partial V_{2}}{\partial e_{d}}\right| \leq \frac{\mu}{\Upsilon}\right\}, \quad \forall \mu>0,
$$

$\left|\frac{\mathrm{d} V_{3}}{\mathrm{~d} x}[0,0, \delta(t)]^{T}\right|$ has an upper bound $\mu U_{T}$.

Thus, the derivative of $V_{3}$ along (17) satisfies

$$
\dot{V}_{3} \leq \dot{\tilde{V}}_{3}+\mu U_{T}
$$

where $\dot{\tilde{V}}_{3}$ is the derivative of $V_{3}$ along (12):

$$
\begin{aligned}
\dot{\tilde{V}}_{3} & =-c_{1}|\omega|^{2 \alpha}+|\omega|^{\alpha} e_{d} \operatorname{sgn}(\omega)+\Upsilon \dot{V}_{2} \\
& \leq-c_{1}|\omega|^{2 \alpha}+|\omega|^{\alpha} e_{d} \operatorname{sgn}(\omega)-\Upsilon \rho_{1} V_{2}^{\frac{2 \alpha}{\alpha+1}} .
\end{aligned}
$$

Clearly, $\dot{\tilde{V}}_{3}$ is homogeneous of 2 with dilation $\left(\frac{1}{\alpha}, \frac{1}{\alpha}, 1\right)$. By virtue of homogeneity technique, the negative definite of $\dot{\tilde{V}}_{3}$ on $\mathbb{R}^{3}$ can be guaranteed by the negative definite on the closed curve

$$
S=\left\{\left(\omega, e_{\omega}, e_{d}\right): \Xi\left(\omega, e_{\omega}, e_{d}\right)=|\omega|^{\alpha}+\left|e_{\omega}\right|^{\alpha}+\left|e_{d}\right|=1\right\} .
$$

Let

$U_{1}=\left\{\left(\omega, e_{\omega}, e_{d}\right) \in \mathbb{R}^{3} \backslash\{0\}:-c_{1}|\omega|^{2 \alpha}+|\omega|^{\alpha} e_{d} \operatorname{sgn}(\omega) \geq 0\right\}$.

Correspondingly, $S_{1}=S \cap U_{1}$ is compact.

Define

$$
N_{1}=\min _{\left(\omega, e_{\omega}, e_{d}\right) \in S_{1}}\left\{\rho_{1} V_{2}^{\frac{2 \alpha}{\alpha+1}}\right\}>0
$$

and

$$
N_{2}=\min _{\left(\omega, e_{\omega}, e_{d}\right) \in S_{1}}\left\{-|\omega|^{\alpha} e_{d} \operatorname{sgn}(\omega)\right\} \leq 0 .
$$

Then, (23) can be written as

$$
\begin{aligned}
-\dot{\tilde{V}}_{3} & \geq c_{1}|\omega|^{2 \alpha}+\Upsilon N_{1}+N_{2} \\
& \geq \Upsilon N_{1}+N_{2} .
\end{aligned}
$$

Hence, $\dot{V}_{3}$ is negative definite when $\Upsilon>-\frac{N_{2}}{N_{1}}$.

Since both $V_{3}$ and $\dot{\tilde{V}}_{3}$ are homogeneous of degrees $1+\frac{1}{\alpha}$ and 2 with dilation $\left(\frac{1}{\alpha}, \frac{1}{\alpha}, 1\right)$, respectively, it follows that

$$
\dot{\tilde{V}}_{3} \leq-\rho_{2} V_{3}^{\frac{2 \alpha}{\alpha+1}}
$$

where $\rho_{2}=-\min _{\left\{\left(\omega, e_{\omega}, e_{d}\right): V_{3}\left(\omega, e_{\omega}, e_{d}\right)=1\right\}}\left\{\dot{V}_{3}>0\right\}$.

Substituting (29) into (22) gives

$$
\dot{V}_{3} \leq-\rho_{2} V_{3}^{\frac{2 \alpha}{\alpha+1}}+\mu U_{T} .
$$

According to $(30)$, when $V_{3} \geq\left(\frac{2 \mu U_{T}}{\rho_{2}}\right)^{\frac{\alpha+1}{2 \alpha}}$, it follows that

$$
\dot{V}_{3} \leq-\frac{1}{2} \rho_{2} V_{3}^{\frac{2 \alpha}{\alpha+1}} \text {. }
$$

By definition, $V_{3}$ is the FTISS-Lyapunov function, and therefore the zero solution of the closed-loop guidance system (17) is locally finite-time input-to-state stable [19].

In particular, we can specialize Theorem 3 to the constant disturbance case:

Corollary 1. Assume $\delta(t)=0$. Then, the zero solution of the closed-loop guidance system (17) is locally finite-time stable.

\subsection{Convergence of LOS rate in the presence of control saturation}

In this subsection, the convergence analysis is conducted in the presence of the control saturation, i.e., when

$$
\mid \phi\left(\lambda, \omega, \hat{d}_{T} \mid \geq U_{M}\right.
$$

The guidance command (10) in this case is replaced by

$$
a_{M}=U_{M} \operatorname{sgn}\left[\phi\left(\lambda, \omega, \hat{d}_{T}\right)\right] .
$$

Substituting (33) into (2), the closed-loop dynamics of the LOS rate is given as

$$
\begin{aligned}
\dot{\omega}= & \frac{-2 \dot{r} \omega}{r}-\frac{\cos \left(\lambda-\gamma_{M}\right) U_{M} \operatorname{sgn}\left[\phi\left(\lambda, \omega, \hat{d}_{T}\right)\right]}{r} \\
& +\frac{\cos \left(\lambda-\gamma_{T}\right) a_{T}}{r},
\end{aligned}
$$

where $\omega(0)=\omega_{0}$.

Assumption 5. $\dot{r}<0,\left|\lambda-\gamma_{M}\right|<\frac{\pi}{2}$, and $\cos (\lambda-$ $\left.\gamma_{M}\right) U_{M}>\left|\cos \left(\lambda-\gamma_{T}\right)\right| U_{T}$.

In light of Assumption 5, $\dot{r}<0$ and $\left|\lambda-\gamma_{M}\right|<\frac{\pi}{2}$ are easily satisfied in a realistic engagement. $\cos \left(\lambda-\gamma_{M}\right) U_{M}>$ $\left|\cos \left(\lambda-\gamma_{T}\right)\right| U_{T}$ means that the maneuverable capability of the interceptor is greater than that of the target.

Next, we establish the corresponding convergence of the LOS rate.

Theorem 3. Under Assumption 5,

1. When $\operatorname{sgn}\left[\phi\left(\lambda, \omega, \hat{d}_{T}\right)\right]=\operatorname{sgn}(\omega)$, for $\left|\omega_{0}\right|<-\frac{L_{a}}{2 L_{\dot{r}}}$, where $L_{a}=\inf _{t \geq 0} \cos \left(\lambda-\gamma_{M}\right) U_{M}-\left|\cos \left(\lambda-\gamma_{T}\right)\right| U_{T}$, and $L_{\dot{r}}=\inf _{t \geq 0} \dot{r},|\omega|$ in system (34) monotonously decreases;

2. When $\operatorname{sgn}\left[\phi\left(\lambda, \omega, \hat{d}_{T}\right)\right]=-\operatorname{sgn}(\omega),|\omega|$ in system $(34)$ is bounded, and $|\omega(t)| \leq \max \left\{\left|\omega_{0}\right|,\left(\frac{\Delta}{c_{1}}\right)^{\frac{1}{\beta}}\right\}$, where $\Delta=\sup _{t \geq 0}\left(\left|e_{d}\right|\right)$.

Proof. Theorem 1 is proved using the positive definite function

$$
V_{4}=\frac{1}{2} \omega^{2}
$$


Consider the derivative of $V_{4}$ along (34)

$$
\begin{aligned}
\dot{V}_{4}= & \frac{-2 \dot{r} \omega^{2}}{r}-\frac{\cos \left(\lambda-\gamma_{M}\right) U_{M} \operatorname{sgn}\left[\phi\left(\lambda, \omega, \hat{d}_{T}\right)\right] \omega}{r} \\
& +\frac{\cos \left(\lambda-\gamma_{T}\right) a_{T} \omega}{r} .
\end{aligned}
$$

By Lyapunov stability theory, the stability of the LOS rate can be guaranteed when $\dot{V}_{4} \leq 0$. The proof proceeds in two cases, namely, $\operatorname{sgn}\left[\phi\left(\lambda, \omega, \hat{d}_{T}\right)\right]=\operatorname{sgn}(\omega)$ and $\operatorname{sgn}\left[\phi\left(\lambda, \omega, \hat{d}_{T}\right)\right]=-\operatorname{sgn}(\omega)$.

\subsection{1. $\operatorname{sgn}\left[\phi\left(\lambda, \omega, \hat{d}_{T}\right)\right]=\operatorname{sgn}(\omega)$}

Substituting

$$
\operatorname{sgn}\left[\phi\left(\lambda, \omega, \hat{d}_{T}\right)\right]=\operatorname{sgn}(\omega)
$$

into (36) gives

$$
\begin{aligned}
\dot{V}_{4} & =\frac{-2 \dot{r} \omega^{2}}{r}-\frac{\cos \left(\lambda-\gamma_{M}\right) U_{M}|\omega|}{r}+\frac{\cos \left(\lambda-\gamma_{T}\right) a_{T} \omega}{r} \\
& \leq \frac{-2 \dot{r} \omega^{2}}{r}-\frac{|\omega|\left[\cos \left(\lambda-\gamma_{M}\right) U_{M}-\left|\cos \left(\lambda-\gamma_{T}\right)\right| U_{T}\right]}{r}
\end{aligned}
$$

Let $\inf _{t \geq 0} \cos \left(\lambda-\gamma_{M}\right) U_{M}-\left|\cos \left(\lambda-\gamma_{T}\right)\right| U_{T}=L_{a}$, and $\inf _{t \geq 0} \dot{r}=L_{\dot{r}}$. According to (38), $\dot{V}_{4} \leq 0$ when the initial LOS rate satisfies $\left|\omega_{0}\right|<-\frac{L_{a}}{2 L_{\dot{r}}}$. Then, using LaSalle's invariance theorem, it is established that $|\omega|$ ceases to decrease as $|\omega|=0$. The $\mathcal{L}_{\infty}$ performance is as follows:

$$
|\omega(t)| \leq\left|\omega_{0}\right|
$$

4.3.2. $\operatorname{sgn}\left[\phi\left(\lambda, \omega, \hat{d}_{T}\right)\right]=-\operatorname{sgn}(\omega)$

Substituting

$$
\operatorname{sgn}\left[\phi\left(\lambda, \omega, \hat{d}_{T}\right)\right]=-\operatorname{sgn}(\omega)
$$

into (36) arrives at

$$
\dot{V}_{4}=\frac{-2 \dot{r} \omega^{2}}{r}+\frac{\cos \left(\lambda-\gamma_{M}\right) U_{M}|\omega|}{r}+\frac{\cos \left(\lambda-\gamma_{T}\right) a_{T} \omega}{r} .
$$

Together with condition $\mid \phi\left(\lambda, \omega, \hat{d}_{T} \mid \geq U_{M}\right.$ and

$$
\operatorname{sgn}\left[\phi\left(\lambda, \omega, \hat{d}_{T}\right)\right]=-\operatorname{sgn}(\omega)
$$

yields

$$
U_{M}|\omega| \leq-\phi\left(\lambda, \omega, \hat{d}_{T}\right) \omega
$$

Substituting expression $\phi\left(\lambda, \omega, \hat{d}_{T}\right)$ into (43) gives

$$
U_{M}|\omega| \leq-\frac{\omega}{\cos \left(\lambda-\gamma_{M}\right)}\left(-2 \dot{r} \omega+c_{1} r|\omega|^{\beta} \operatorname{sgn}(\omega)+r \hat{d}_{T}\right) .
$$

By manipulation, (44) can be rewritten as

$$
\begin{aligned}
\frac{-2 \dot{r} \omega^{2}}{r} & +\frac{\cos \left(\lambda-\gamma_{M}\right) U_{M}|\omega|}{r}+\frac{\cos \left(\lambda-\gamma_{T}\right) a_{T} \omega}{r} \\
& +c_{1}|\omega|^{1+\beta}-\left(d_{T}-\hat{d}_{T}\right) \omega \leq 0
\end{aligned}
$$

which is equivalent to

$$
\begin{aligned}
\dot{V}_{4} & \leq-c_{1}|\omega|^{1+\beta}+e_{d} \omega \\
& \leq-c_{1}|\omega|^{1+\beta}+\Delta|\omega|
\end{aligned}
$$

where $\Delta=\sup _{t \geq 0}\left(\left|e_{d}\right|\right)$. Therefore, to ensure $\dot{V}_{4} \leq 0,|\omega|$ needs to satisfies $|\omega| \geq\left(\frac{\Delta}{c_{1}}\right)^{\frac{1}{\beta}}$. At a result, $|\omega|$ will be bounded within $\left(\frac{\Delta}{c_{1}}\right)^{\frac{1}{\beta}}$. The $\mathcal{L}_{\infty}$ performance in this case is as follows:

$$
|\omega(t)| \leq \max \left\{\left|\omega_{0}\right|,\left(\frac{\Delta}{c_{1}}\right)^{\frac{1}{\beta}}\right\} .
$$

In fact, this case represents an engagement wherein the target maneuver is beneficial to the interceptor.

Remark 5. In the maneuverable target interception literature, there are only a few works that exploit the control saturation and rigorously analyze the resulting transient performance. In [7], an auxiliary low-pass filter is added to compensate for effect of the control saturation. A fast adaptive guidance law in [11] induces an auxiliary signal to prevent the control saturation from destroying the parameter adaptation. The objective of dealing with the control saturation in these two guidance laws is to enable the adaptive laws to work so that the parameters of the target maneuvers can be effectively estimated. Since the resulting design is closely coupled with the adaptive laws, both of them do not provide explicit convergence properties of the $L O S$ rate in the presence of the control saturation, particularly regarding the transient performance. Different from these two guidance laws, the RFTG law directly use the control saturation to drive the LOS rate to a sufficiently small neighborhood of the origin, and the estimation of the effect of the target maneuvers does not be affected by the control saturation due to the design of CFTDO.

Remark 6. A theoretical proof of convergence of the LOS rate in this case is very challenging. To the best knowledge of the authors, this is the first attempt to rigorously analyze the convergence property in the presence of the control saturation. Admittedly, a number of assumptions are made for the system states in Theorem 3. Without the assumptions in Theorem 3, it is hard to ensure the convergence of the LOS rate. In fact, the assumptions employed in Theorem 3 have clearly physical meanings and can be easily satisfied in practice, which is illustrated in the numerical evidence in the next section.

Remark 7. When the control saturation happens and the engagement conditions satisfy the assumptions in Theorem 3 , the LOS rate will decrease in magnitude, thus reducing $\mid \phi\left(\lambda, \omega, \hat{d}_{T} \mid\right.$. Once $\mid \phi\left(\lambda, \omega, \hat{d}_{T} \mid<U_{M}\right.$, the system trajectories will enter the linear region of the RFTG law, and correspondingly, the convergence property will follows Theorem 2. 


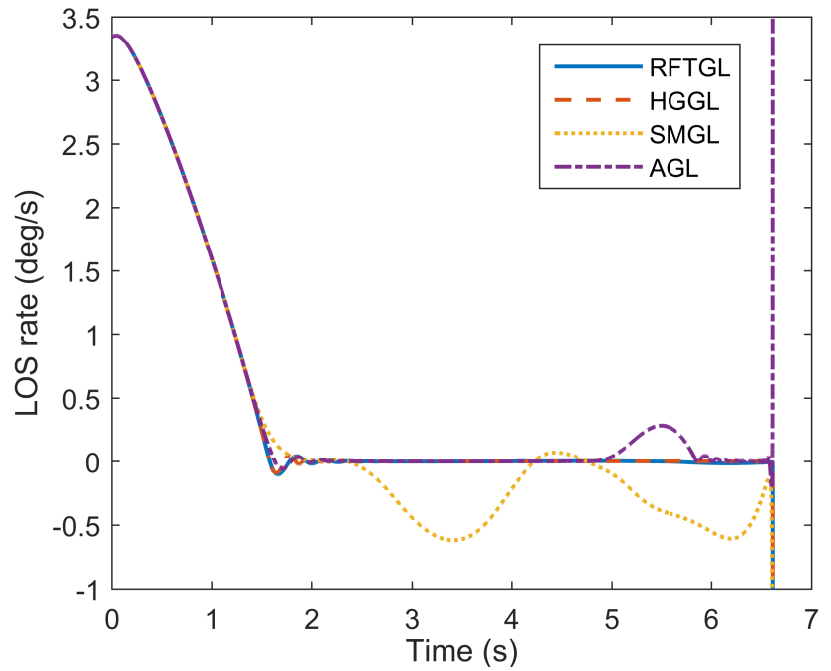

(a)

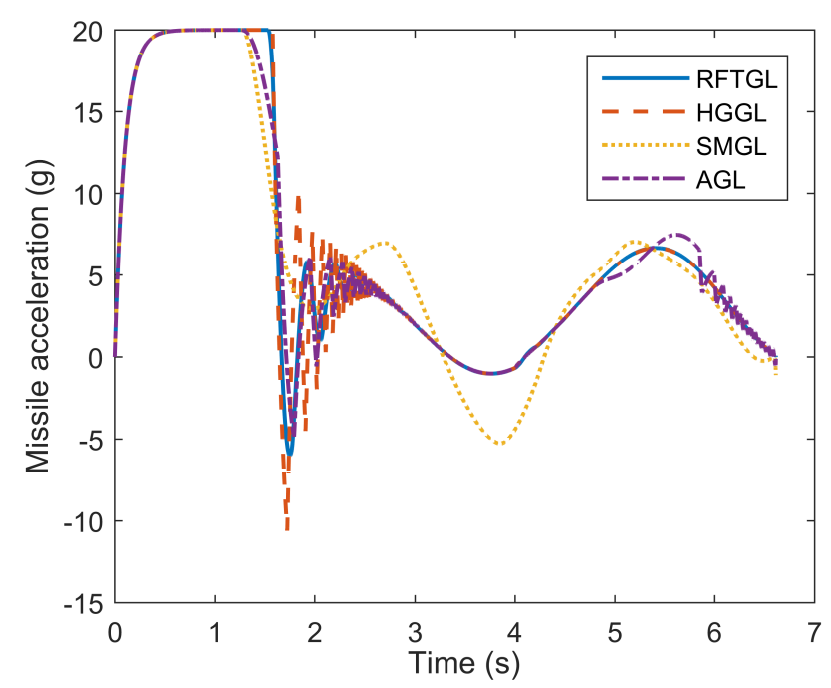

(c)

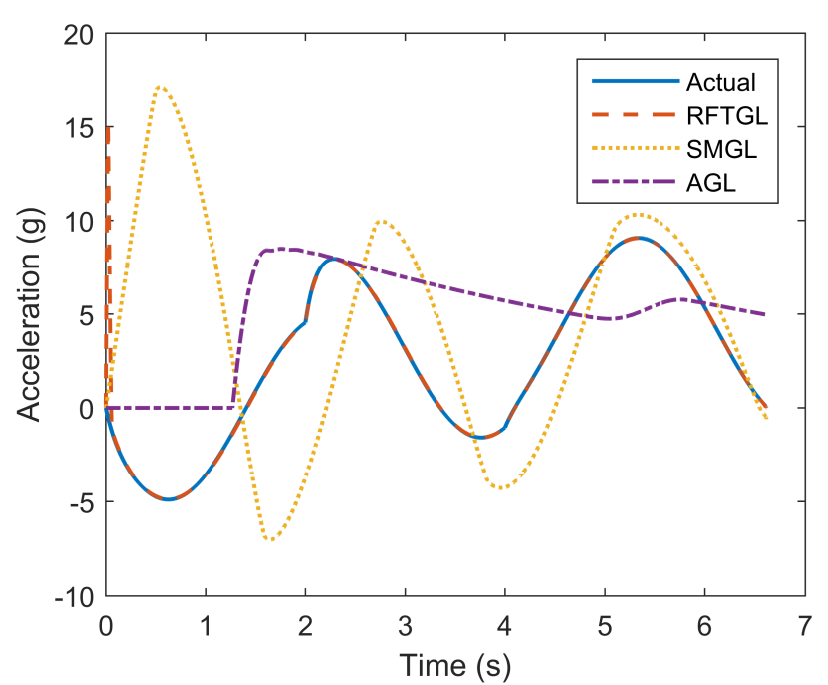

(b)

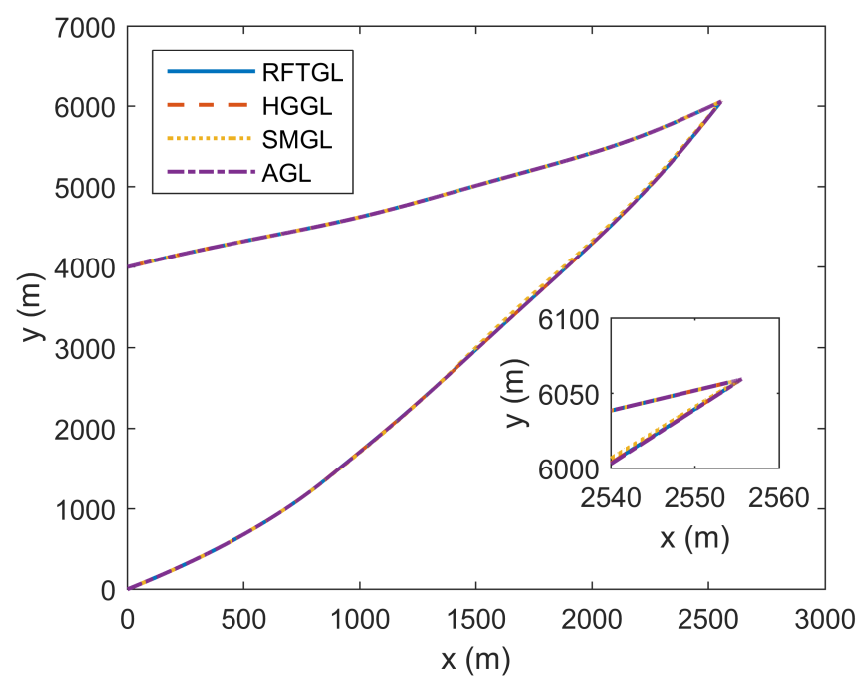

(d)

Fig. 1. Simulation results for MS-GLs.

\section{Simulations}

In this section, numerical comparison results for the RFTG law and the other three guidance laws are presented. Based on a target maneuver model of the UMTI, case studies are also carried out where guidance performance is systematically assessed for the proposed RFTG law.

\subsection{A target maneuver model of UMTI}

In accordance to the general model (6), a target maneuver model is given as follows:

$$
\iota \dot{a}_{T}+a_{T}=\Xi_{1}(\omega)+\Xi_{2}(\eta)+\Xi_{3}(\varpi),
$$

where $\iota$ is the time constant, and

$$
\begin{aligned}
\Xi_{1}(\omega) & =U_{T}^{\Xi_{1}} \operatorname{sgn}\left[\cos \left(\lambda-\gamma_{T}\right) \omega\right], \\
\Xi_{2}(\eta) & =C \eta, \\
\dot{\eta} & =\Lambda \eta, \\
\Xi_{3}(\varpi) & =U_{T}^{\Xi_{3}} \tanh (\varphi \varpi),
\end{aligned}
$$

where $U_{T}^{\Xi_{1}}$, and $U_{T}^{\Xi_{3}}$ are two positive constants. $\eta_{0}$ is the initial state of $\eta$. $\Lambda$ is a $n \times n$ constant coefficient matrix with all eigenvalues lying on the imaginary axis. $C$ is a constant vector of suitable size. $\tanh (\cdot)$ is a hyperbolic tangent function. $\varpi>0 . \varphi>0$ is the parameter regarding the slope of $\Xi_{3}(\varpi)$ at $\varpi=0$.

In view of (48), $\Xi_{1}(\omega)$ is the evasive maneuver which aims to increase $|\omega|$ and zero-effort-miss (ZEM); $\Xi_{2}(\eta)$ is the periodic maneuver that is composed of sinusoidal maneuvers; $\Xi_{3}(\varpi)$ is the so-called deterministic yet unknown 
maneuver which is determined by a combination of two step functions:

$$
\varpi= \begin{cases}0, & 0 \leq t \leq t_{1} \\ \nu_{1}, & t_{1}<t<t_{2} \\ \nu_{2}, & t \geq t_{2}\end{cases}
$$

\subsection{Comparisons with other MS-GLs}

For the sake of comparison, three MS-GLs are chosen as follows:

\subsubsection{Adaptive guidance law}

The adaptive guidance law (AGL) is adapted from [7]:

$$
\begin{aligned}
a_{M} & =-N_{a} \dot{r} \omega+k_{a} r \omega+\hat{d}_{a} \tanh \left(\frac{r \omega}{\varepsilon}\right), \\
\dot{\hat{d}}_{a} & =\sigma r \omega \tanh \left(\frac{r \omega}{\varepsilon}\right)+\sigma \varepsilon-\sigma \zeta \hat{d}_{a},
\end{aligned}
$$

where $N_{a}>1 . \quad k_{a}>0 . \quad \sigma>0$ is the adaptation gain. $\varepsilon>0 . \quad \zeta>0$. Note that, compared with the adaptive guidance law in [7], the AGL used here does not explicitly consider the second-order autopilot dynamics. In a realistic short-range engagement, the autopilot dynamics of the interceptor are nonlinear and time-varying, particularly regarding the highly maneuverable targets. In such a situation, the time-invariant second-order linear system used in [7] is not proper [32].

\subsubsection{Sliding mode guidance law}

Let the sliding surface be $\Omega=r \omega-c_{s} \sqrt{r}$. The sliding mode guidance law (SMGL) consists of the second-order sliding mode observer (SOSMO) and the second-order sliding mode stabilizer (SOSMS) [14]:

$$
\begin{gathered}
a_{M}=\frac{1}{\cos \left(\lambda-\gamma_{M}\right)}\left(\zeta_{1}|\Omega|^{2 / 3} \operatorname{sgn}(\Omega)+\zeta_{2} \int|\Omega|^{1 / 3} \operatorname{sgn} \Omega \mathrm{d} t\right. \\
\left.-N_{s} \dot{r} \omega-c_{s} \dot{r} /(2 \sqrt{r})+z_{1}\right),
\end{gathered}
$$

where $z_{1}$ is obtained by the following observer:

$$
\begin{aligned}
& \dot{z}_{0}=v_{0}-\cos \left(\lambda-\gamma_{M}\right) a_{M_{\gamma}}-\dot{r} \omega-c_{s} \dot{r} /(2 \sqrt{r}), \\
& \dot{z}_{1}=v_{1}, \\
& \dot{z}_{2}=-1.1 L \operatorname{sgn}\left(z_{2}-v_{1}\right),
\end{aligned}
$$

in which

$$
\begin{aligned}
& v_{0}=-2 L^{1 / 3}\left|z_{0}-\Omega\right|^{2 / 3} \operatorname{sgn}\left(z_{0}-\Omega\right)+z_{1}, \\
& v_{1}=-1.5 L^{1 / 2}\left|z_{1}-v_{0}\right|^{1 / 2} \operatorname{sgn}\left(z_{1}-v_{0}\right)+z_{2} .
\end{aligned}
$$

It is noted that $L$ is the Lipschitz constant, determined by the upper bound of the derivative of the target acceleration.
Table 1. Summary of homing accuracy

\begin{tabular}{lc}
\hline Method & Miss distance $(\mathrm{m})$ \\
\hline RFTGL & 0.00002 \\
AGL & 0.00011 \\
SMGL & 0.00029 \\
HGGL & 0.00027 \\
\hline
\end{tabular}

\subsubsection{High-gain guidance law}

The high-gain guidance law (HGGL) is used to suppress the effect of the target maneuvers [33]:

$$
a_{M}=\frac{1}{\cos \left(\lambda-\gamma_{M}\right)}\left(-2 \dot{r} \omega+c_{h} r|\omega|^{\beta_{h}} \operatorname{sgn}(\omega)+\frac{r \omega}{2 \Theta^{2}}\right)
$$

where the design parameter $\Theta$ is determined by the upper bound of $\left|a_{T}\right|$. Considering the unpredictable targets, $\Theta$ needs to be chosen sufficiently small to guarantee a predetermined ultimate bound of $|\omega|$ throughout intercept. The parameters of the RFTG law are chosen as $c_{1}=2, \alpha=0.6$, and $k_{1}=k_{2}=5$. The parameters of the AGL are chosen as $N_{a}=4, k_{a}=3, \sigma=10, \varepsilon=0.02$, and $\zeta=0.02$. The parameters of the SMGL are chosen as $L=100, c_{s}=0$, $N=6$, and $\zeta_{1}=\zeta_{2}=20$. The parameters of the HGGL are chosen as $c_{h}=2, \beta=0.6$, and $\Upsilon=0.01$.

In the simulation, the interceptor's initial position is set at the origin, its initial velocity is $1000 \mathrm{~m} / \mathrm{s}$, and its initial flight path angle is $50 \mathrm{deg}$. The attitude dynamics of the interceptor is modeled as a first-order system with a time constant $0.1 \mathrm{~s}$. The target's initial position coordinates are $x_{T_{0}}=0 \mathrm{~m}$, and $y_{T_{0}}=4000 \mathrm{~m}$, its initial velocity is $500 \mathrm{~m} / \mathrm{s}$, and its initial flight path angle is $35 \mathrm{deg}$. The parameters of the target maneuver model (48) are chosen as $\iota=0.1 \mathrm{~s}$, $U_{T}^{\Xi_{1}}=0 \mathrm{~m} / \mathrm{s}^{2}, C=\left[\begin{array}{ll}1 & 0\end{array}\right], \eta_{0}=\left[\begin{array}{ll}-23.8 & 42.4\end{array}\right]^{\top} \mathrm{m} / \mathrm{s}^{2}$, $\Lambda=\left[\begin{array}{cc}0 & -2 \\ 2 & 0\end{array}\right], U_{T}^{\Xi_{3}}=47.5 \mathrm{~m} / \mathrm{s}^{2}, \varphi=0.2, t_{1}=2 \mathrm{~s}, t_{2}=4 \mathrm{~s}$, $\nu_{1}=4$, and $\nu_{2}=6$. Note that we select $U_{T}^{\Xi_{1}}=0 \mathrm{~m} / \mathrm{s}^{2}$ to render the comparison in the same setting.

Figure 1a presents the LOS rates of the four guidance laws. As shown, the LOS rates of the four guidance laws diverge at last. It is worth mentioning that this divergence phenomenon cannot be avoided due to the inherent property of the effect of the disturbance. The convergence of the RFTG law and the HGGL is better than that of the AGL and the SMGL. This can be attributed to the manifolds used in guidance design: the RFTG law and the HGGL directly employ the LOS rate as the manifold, while the AGL and the SMGL use the transversal component of the relative velocity. In addition, the divergence of the AGL that begins at approximately $5 \mathrm{~s}$ is caused by the parameter adaption. Figure 1b describes the disturbance observation performance of the RFTG law, the SMGL, and the AGL. Compared with the SMGL, and the AGL, the CFTDO of the RFTG law can estimate the disturbance with a faster convergence rate and greater precision. The performance deterioration of the SMGL is caused by the improper choice of the critical design parameter, $L$, which 
is closely related to the derivative of the target acceleration. The upper bound adaption in the AGL also stops at the beginning of the intercept, so as to avoid adverse effects of control saturation. As depicted in Figs. 1a and 1b, there exists a conflict between the adaption process and the stabilization: the adaption dose not work when the stabilization error is relatively small. Figure 1c gives the guidance commands generated by the four methods. The profile of the RFTG law is smooth. The profile of the HGGL oscillates severely due to the high gain used. The profile of the SMGL is conservative because of the estimated disturbance. The profile of the AGL has a large number of undesired small spikes, which is mainly caused by the adaption process. Figure 1d depicts the flight trajectories, and, correspondingly, table 1 gives the homing accuracy of the four guidance laws, demonstrating homing precision of the RFTG law is better than that of the other three in the presence of the target maneuver.

\subsection{Guidance performance analysis for RFTG Law}

In the subsection, four cases are studied to illustrate the guidance performance regarding the initial LOS rate, $\Xi_{1}(\omega), \Xi_{2}(\eta)$, and $\Xi_{3}(\varpi)$, respectively. The parameters of the RFTG law are chosen as $U_{M}=196 \mathrm{~m} / \mathrm{s}^{2}, c_{1}=1$, $\alpha=0.6$, and $k_{1}=k_{2}=100$.

The tests in the simulations are compared with the nominal case. In light of the nominal case, the interceptor's initial position is set at the origin, its initial velocity is $1000 \mathrm{~m} / \mathrm{s}$, and its initial flight path angle is $65 \mathrm{deg}$. The attitude dynamics of the interceptor is modeled as a firstorder system with a time constant $0.1 \mathrm{~s}$. The target's initial position coordinates are $x_{T_{0}}=4 \mathrm{~km}$, and $y_{T_{0}}=0 \mathrm{~km}$, its initial velocity is $500 \mathrm{~m} / \mathrm{s}$, and its initial flight path angle is $35 \mathrm{deg}$. The parameters of the target maneuver model are chosen as $\iota=0.1 \mathrm{~s}, U_{T}^{\Xi_{1}}=0 \mathrm{~m} / \mathrm{s}^{2}, C=\left[\begin{array}{ll}1 & 0\end{array}\right]$, $\eta_{0}=\left[\begin{array}{ll}39.2 & 39.2\end{array}\right]^{\top} \mathrm{m} / \mathrm{s}^{2}, \Lambda=\left[\begin{array}{cc}0 & -1 \\ 1 & 0\end{array}\right], U_{T}^{\Xi_{3}}=98 \mathrm{~m} / \mathrm{s}^{2}$, $\varphi=0.2, t_{1}=2 \mathrm{~s}, t_{2}=4 \mathrm{~s}, \nu_{1}=4$, and $\nu_{2}=10$.

\subsubsection{The initial LOS rate}

Two interception scenarios, together with the nominal case, are employed to demonstrate the robustness of the RFTG law in term of the initial LOS rate. For the two interception scenarios, referred to as Case 1 and Case 2, the initial flight-path angles are $\gamma_{0}=45 \mathrm{deg}$ in Case 1 , and $\gamma_{0}=85 \mathrm{deg}$ in Case 2, and the other parameters are the same as that of the nominal case. The miss-distances of the nominal case, Case 1, and Case 2 are $0.0143 \mathrm{~m}, 0.0106 \mathrm{~m}$, and $0.0136 \mathrm{~m}$, respectively. The miss-distances in these three cases are high-precision, thus demonstrating the satisfactory robustness of the RFTG law to the initial LOS rate. Figure 2 a presents the LOS rates of the three cases, and Fig. 2b shows the corresponding missile and the target acceleration. From the two figures, it can be observed that the control saturation occurs in Case 1 and Case 2 at the beginning of the intercept, while the LOS rates quickly converge to the origin. Such control saturation is caused by the large magnitude of the initial LOS rates. The homing accuracy in Case 1 and Case 2, in fact, illustrates the effectiveness of the RFTG law in the presence of control saturation, and Theorem 1 has stated this result. Besides, as shown in Fig. 2b, the missile acceleration is mainly used to compensate for the effect of the target maneuver when the LOS rates in magnitude are relatively small.

\subsubsection{Target maneuver $\Xi_{1}(\omega)$}

Two interception cases, Case 3 and Case 4 , together with the nominal case, are employed to demonstrate the guidance performance with respect to the target maneuver $\Xi_{1}(\omega)$. In light of the two interception cases, the parameters of interest are chosen as $U_{T}^{\Xi_{1}}=49 \mathrm{~m} / \mathrm{s}^{2}$ in Case 3 and $U_{T}^{\Xi_{1}}=24.5 \mathrm{~m} / \mathrm{s}^{2}$ in Case 4 ; the other simulation parameters are the same as that of the nominal case. The miss-distances of the nominal case, Case 3, and Case 4 are $0.0143 \mathrm{~m}, 0.7170 \mathrm{~m}$, and $0.0607 \mathrm{~m}$, respectively. Obviously, compared with the nominal case, the homing accuracy of Case 3 deteriorates significantly. As described in Fig. 2c, the main reason is the large oscillations of the LOS rates when the interceptor approaches the target closely. These oscillations are induced by the rapidly varying effect of the target maneuver $\Xi_{1}(\omega)$, which is enlarged by the decreasing interceptor-target range. In addition, note that, at the start of the intercept, the LOS rates are rendered within small errors by virtue of the non-smooth feedback when the interceptor-target range is relatively large.

\subsubsection{Target maneuver $\Xi_{2}(\eta)$}

Two interception engagements, together with the nominal case, are used to assess the guidance performance against the weaving targets with different frequencies. Given these two interception engagements, which are referred to as Case 5 and Case 6, the parameters in question are set for $\Lambda=\left[\begin{array}{cc}0 & -1.5 \\ 1.5 & 0\end{array}\right]$ in Case 5 , and $\Lambda=\left[\begin{array}{cc}0 & -0.5 \\ 0.5 & 0\end{array}\right]$ in Case 6 , and the other parameters in the simulations are the same as that of the nominal case. The homing accuracy of these three cases is high: the miss-distances of the nominal case, Case 5, and Case 6 are $0.0143 \mathrm{~m}, 0.0131 \mathrm{~m}$, and $0.0042 \mathrm{~m}$, respectively. As depicted in Fig. 3a, the LOS rates are stabilized well in the presence of the sinusoidal maneuvers with $0.5 \mathrm{rad} / \mathrm{s}, 1 \mathrm{rad} / \mathrm{s}$, and $1.5 \mathrm{rad} / \mathrm{s}$. Such three cases demonstrate that the RFTG law can effectively deal with the weaving targets.

\subsubsection{Target maneuver $\Xi_{3}(\varpi)$}

Two interception cases, together with the nominal case, are adopted to illustrate the guidance performance in term of the so-called deterministic yet unknown maneuvers. With respect to these two interception cases, which are referred to as Case 7 and Case 8, the parameters of interest are chosen as $t_{2}=5 \mathrm{~s}$ in Case 7 , and $U_{T}^{\Xi_{3}}=147 \mathrm{~m} / \mathrm{s}^{2}$ in Case 8 , the other parameters used in the simulations are the same as that of the nominal case. The miss-distances of the 


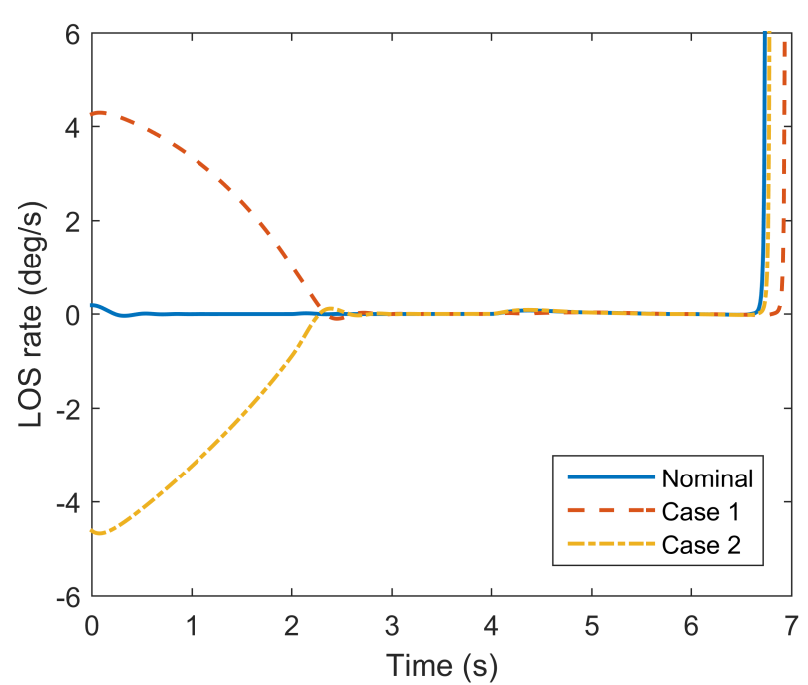

(a)

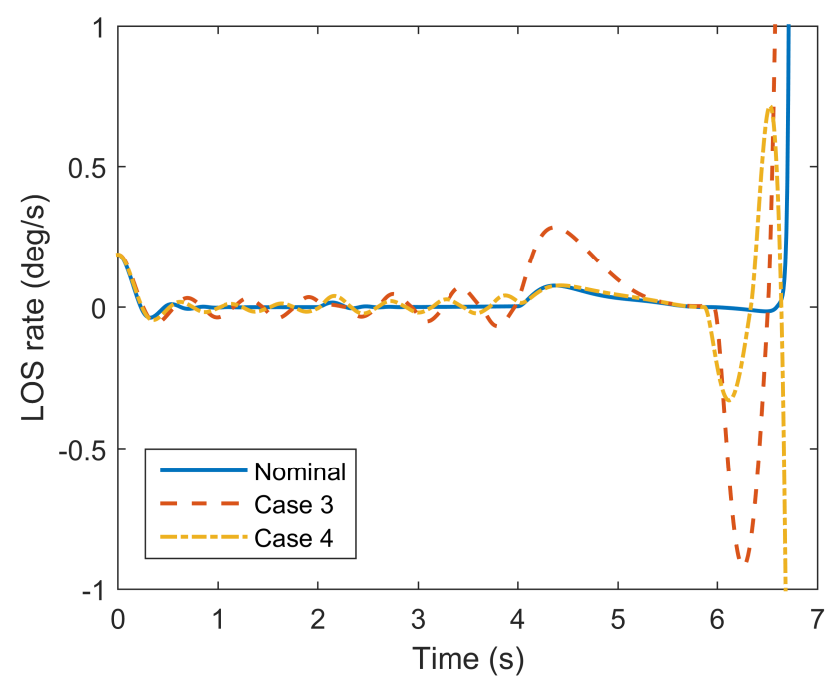

(c)

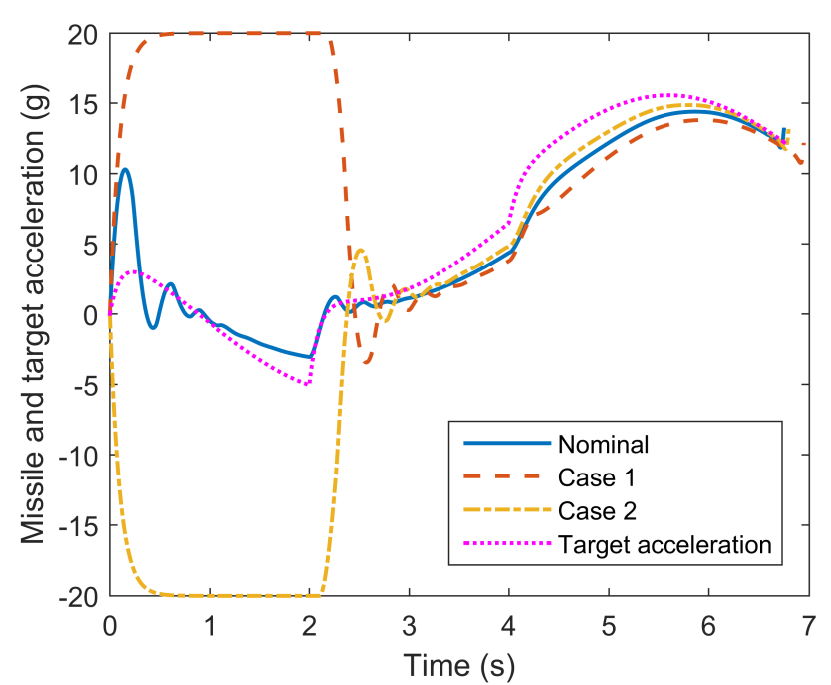

(b)
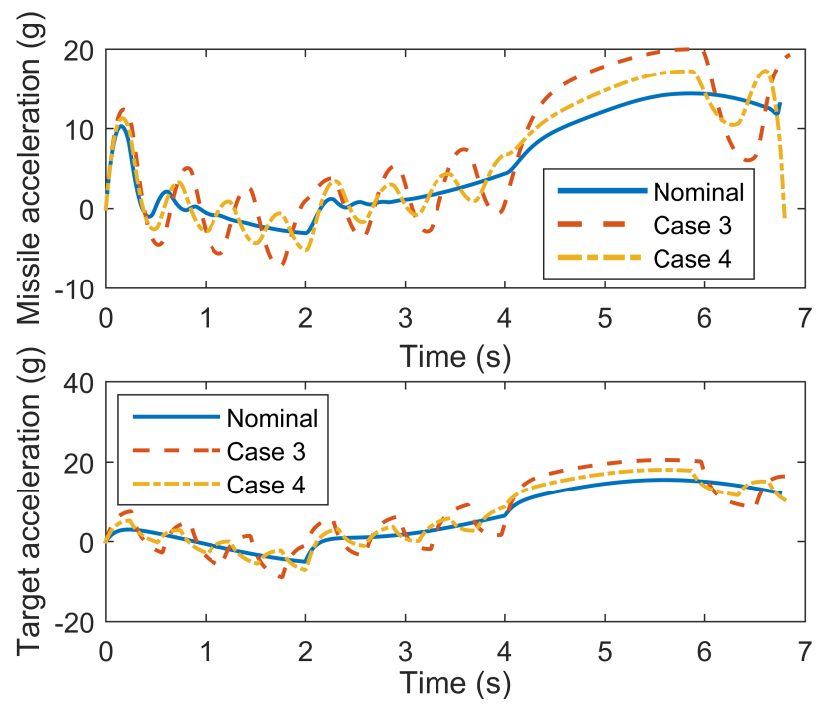

(d)

Fig. 2. Simulation results for Cases 1, 2, 3 and 4 .

nominal case, Case 7, and Case 8 are $0.0143 \mathrm{~m}, 0.0146 \mathrm{~m}$, and $0.0276 \mathrm{~m}$, respectively. As described in Figs. 3c and 3d, the LOS rate in Case 7 is quickly nullified after the sudden change of the target maneuver at $5 \mathrm{~s}$, while the LOS rate in Case 8 is stabilized through a relative large transient period due to the control saturation, which is the adverse impact of the large magnitude of the target acceleration. Furthermore, because the maneuverability of the target is larger than that of the interceptor at about $5.5 \mathrm{~s}$ to $6.0 \mathrm{~s}$, the divergence of the LOS rate in Case 8 lasts a short period of time.

\section{Conclusion}

In this paper, the integrated continuous finite-time disturbance observer (CFTDO)/bounded continuous finitetime stabilizer (BCFTS) strategy is proposed to approach the problem of intercepting a maneuvering target with an unpredictable evasive strategy. The local FTISS of the proposed RFTG law is established in the case of the bounded derivative of the effect of the target maneuver. Numerical comparisons demonstrate the favorable guidance performance of the RFTG law in terms of the homing accuracy and the estimation of the effect of the target maneuver. Furthermore, the RFTG law can effectively stabilizes the LOS rate in the presence of the control saturation, and achieve the high-precision miss-distances in the four typical cases of the unpredictable maneuverable target interception.

\section{References}

[1] P. Zarchan, Representation of realistic evasive maneuvers by the use of shaping filters, Journal of Guidance, Control, and 


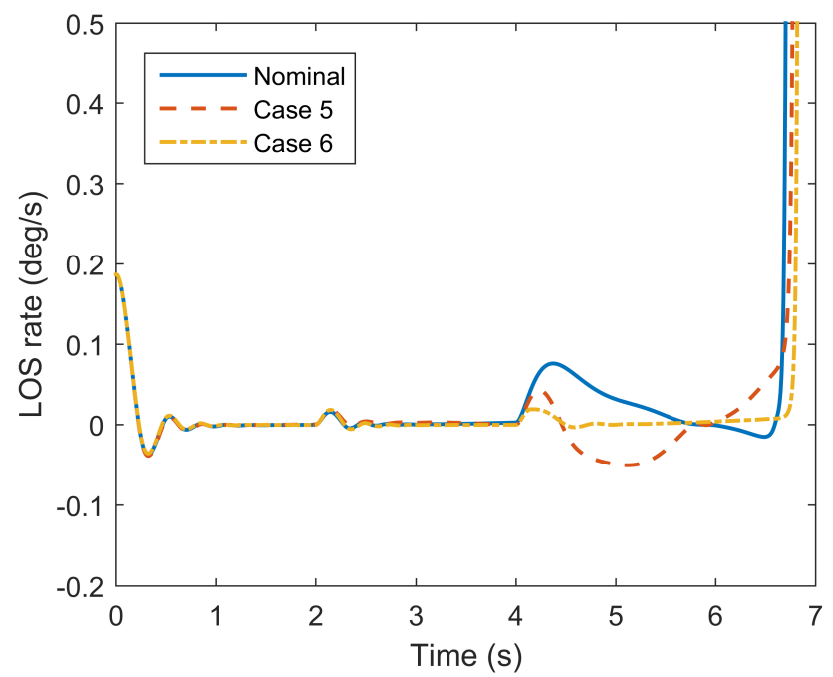

(a)

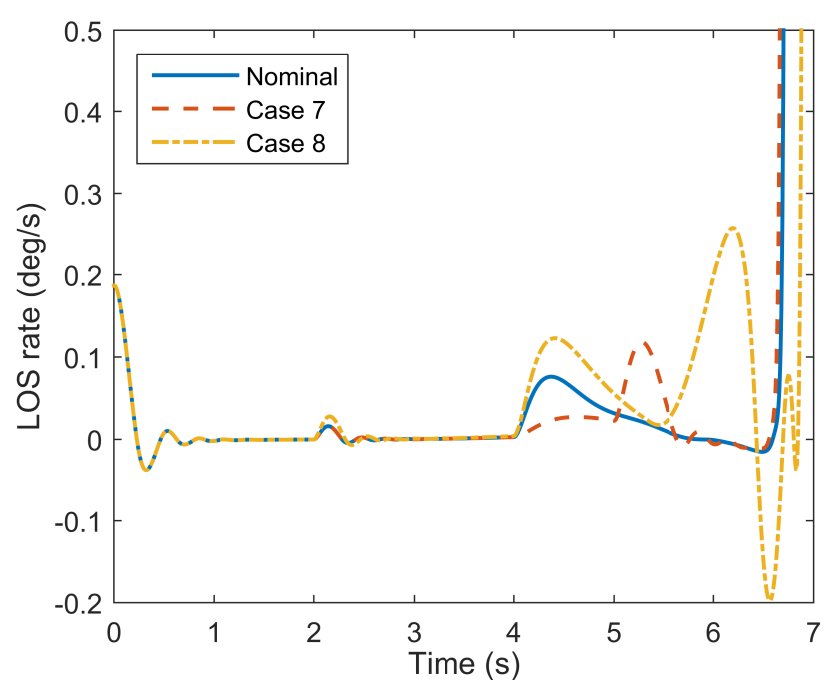

(c)

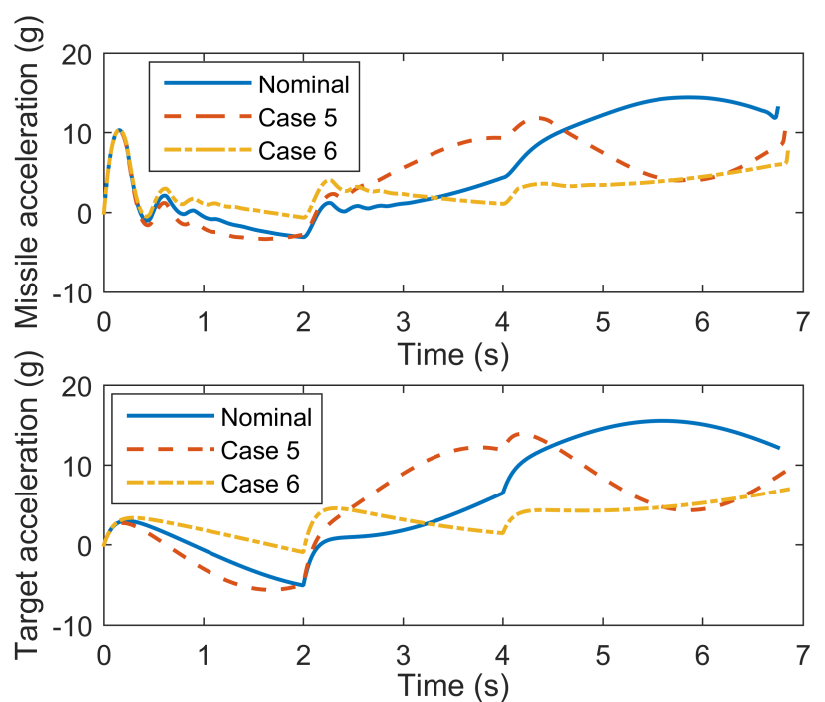

(b)
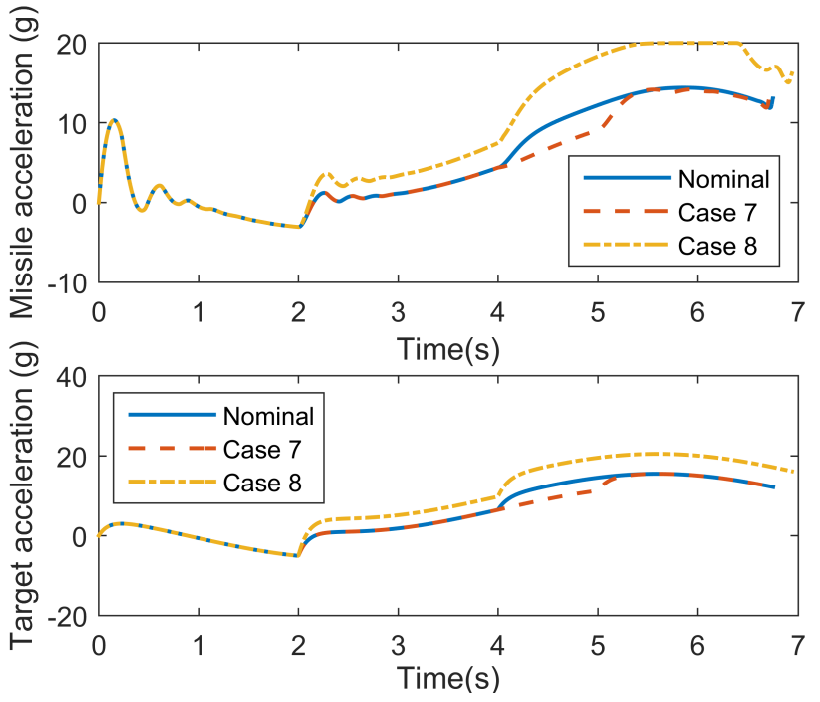

(d)

Fig. 3. Simulation results for Cases 5, 6, 7 and 8 .

Dynamics 2 (4) (1979) 290-295. doi:10.2514/3.55877.

[2] R. Atir, G. Hexner, H. Weiss, T. Shima, Target maneuver adaptive guidance law for a bounded acceleration missile, Journal of Guidance, Control, and Dynamics 33 (3) (2010) 695-706. doi:10.2514/1.47276.

[3] V. Turetsky, T. Shima, Target evasion from a missile performing multiple switches in guidance law, Journal of Guidance, Control, and Dynamics 39 (10) (2016) 2364-2373. doi: 10.2514/1.g000461.

[4] S. Gutman, S. Rubinsky, 3d-nonlinear vector guidance and exoatmospheric interception, IEEE Transactions on Aerospace and Electronic Systems 51 (4) (2015) 3014-3022. doi:10.1109/ taes.2015.140204.

[5] R. H. Chen, J. L. Speyer, D. Lianos, Homing missile guidance and estimation under agile target acceleration, Journal of Guidance, Control, and Dynamics 30 (6) (2007) 1577-1589. doi:10.2514/1.30107.

[6] I. Rusnak, L. Peled-Eitan, Guidance law against spiraling target, Journal of Guidance, Control, and Dynamics 39 (7) (2016) 1694-1696. doi:10.2514/1.g001646.

[7] D. Zhou, B. Xu, Adaptive dynamic surface guidance law with input saturation constraint and autopilot dynamics, Journal of Guidance, Control, and Dynamics 39 (5) (2016) 1155-1162. doi:10.2514/1.g001236.

[8] R. Du, K. Meng, D. Zhou, J. Liu, Design of three-dimensional nonlinear guidance law with bounded acceleration command, Aerospace Science and Technology 46 (2015) 168-175. doi: 10.1016/j.ast.2015.07.010.

[9] W. Wang, S. Xiong, S. Wang, S. Song, C. Lai, Three dimensional impact angle constrained integrated guidance and control for missiles with input saturation and actuator failure, Aerospace Science and Technology 53 (2016) 169-187. doi: $10.1016 /$ j.ast . 2016.03.015

[10] V. Behnamgol, A. R. Vali, A. Mohammadi, A new adaptive finite time nonlinear guidance law to intercept maneuvering targets, Aerospace Science and Technology 68 (2017) 416-421. doi:10.1016/j.ast.2017.05.033.

[11] D. Cho, H. J. Kim, M.-J. Tahk, Fast adaptive guidance against highly maneuvering targets, IEEE Transactions on Aerospace and Electronic Systems 52 (2) (2016) 671-680. doi:10.1109/ taes.2015.140958.

[12] D. Zhou, S. Sun, K. L. Teo, Guidance laws with finite time con- 
vergence, Journal of Guidance, Control, and Dynamics 32 (6) (2009) 1838-1846. doi:10.2514/1.42976.

[13] Y.-Y. Chen, Robust terminal guidance law design for missiles against maneuvering targets, Aerospace Science and Technology 54 (2016) 198-207. doi:10.1016/j.ast.2016.03.028.

[14] Y. B. Shtessel, I. A. Shkolnikov, A. Levant, Smooth secondorder sliding modes: Missile guidance application, Automatica 43 (8) (2007) 1470-1476. doi:10.1016/j.automatica.2007.01. 008.

[15] V. Utkin, Discussion aspects of high-order sliding mode control, IEEE Transactions on Automatic Control 61 (3) (2016) 829833. doi:10.1109/tac.2015.2450571.

[16] J. Zhou, J. Yang, Smooth sliding mode control for missile interception with finite-time convergence, Journal of Guidance, Control, and Dynamics 38 (7) (2015) 1311-1318. doi:10.2514/ 1. g000912.

[17] H. Yan, H.-B. Ji, Guidance laws based on input-to-state stability and high-gain observers, IEEE Transactions on Aerospace and Electronic Systems 48 (3) (2012) 2518-2529. doi:10.1109/ taes.2012.6237606

[18] H.-S. Shin, A. Tsourdos, K.-B. Li, A new three-dimensional sliding mode guidance law variation with finite time convergence, IEEE Transactions on Aerospace and Electronic Systems 53 (5) (2017) 2221-2232. doi:10.1109/taes.2017.2689938.

[19] Y. Hong, Z.-P. Jiang, G. Feng, Finite-time input-to-state stability and applications to finite-time control design, SIAM Journal on Control and Optimization 48 (7) (2010) 4395-4418. doi:10.1137/070712043.

[20] S. Bhat, D. Bernstein, Finite-time stability of homogeneous systems, in: Proceedings of the 1997 American Control Conference (Cat. No.97CH36041), IEEE, 1997. doi:10.1109/acc. 1997.609245

[21] M. Golestani, I. Mohammadzaman, A. R. Vali, Finite-time convergent guidance law based on integral backstepping control, Aerospace Science and Technology 39 (2014) 370-376. doi:10.1016/j.ast.2014.09.018.

[22] C. Li, Z. Qu, Distributed finite-time consensus of nonlinear systems under switching topologies, Automatica 50 (6) (2014) 1626-1631. doi:10.1016/j.automatica.2014.04.002.

[23] S. Li, H. Sun, J. Yang, X. Yu, Continuous finite-time output regulation for disturbed systems under mismatching condition, IEEE Transactions on Automatic Control 60 (1) (2015) 277282. doi:10.1109/tac.2014.2324212.

[24] S. He, D. Lin, J. Wang, Continuous second-order sliding mode based impact angle guidance law, Aerospace Science and Technology 41 (2015) 199-208. doi:10.1016/j.ast.2014.11.020.

[25] N. Wang, C. Qian, J.-C. Sun, Y.-C. Liu, Adaptive robust finitetime trajectory tracking control of fully actuated marine surface vehicles, IEEE Transactions on Control Systems Technology 24 (4) (2016) 1454-1462. doi:10.1109/tcst.2015.2496585.

[26] Y. Si, S. Song, Adaptive reaching law based three-dimensional finite-time guidance law against maneuvering targets with input saturation, Aerospace Science and Technology 70 (2017) 198210. doi:10.1016/j.ast.2017.08.006.

[27] P. Zarchan, Tactical and strategic missile guidance, American Institute of Aeronautics and Astronautics, 2012.

[28] N. Harl, S. N. Balakrishnan, Impact time and angle guidance with sliding mode control, IEEE Transactions on Control Systems Technology 20 (6) (2012) 1436-1449. doi:10.1109/tcst. 2011.2169795.

[29] T. Kailath, Linear systems, Vol. 156, Prentice-Hall Englewood Cliffs, NJ, 1980.

[30] L. Rosier, Homogeneous lyapunov function for homogeneous continuous vector field, Systems \& Control Letters 19 (6) (1992) 467-473. doi:10.1016/0167-6911 (92) 90078-7.

[31] E. F. Beckenbach, R. Bellman, Inequalities, Vol. 30, Springer Science \& Business Media, 2012.

[32] N. Lechevin, C. A. Rabbath, Robust discrete-time proportionalderivative navigation guidance, Journal of Guidance, Control, and Dynamics 35 (3) (2012) 1007-1013. doi:10.2514/1.55783.

[33] G. Li, H. Ji, A three-dimensional robust nonlinear termi- nal guidance law with ISS finite-time convergence, International Journal of Control 89 (5) (2015) 938-949. doi:10.1080/ 00207179.2015 .1107193

\section{Appendix}

\subsection{Appendix 1: homogeneous systems}

Consider dilation $\left(r_{1}, \ldots, r_{n}\right), r_{i}>0, i=1, \ldots, n$, where $\varepsilon=e^{t}, t \in \mathbb{R}$. Then, a real-valued function, $f(x)$, is homogeneous of degree $\vartheta \in \mathbb{R}$ with $\left(r_{1}, \ldots, r_{n}\right)$ if

$$
f\left(\varepsilon^{r_{1}} x_{1}, \ldots, \varepsilon^{r_{n}} x_{n}\right)=\varepsilon^{\vartheta} f(x) .
$$

A vector field, $g(x)=\left[g_{1}(x), \ldots, g_{n}(x)\right]^{\top}$, is homogeneous of degree $p \in \mathbb{R}$ with $\left(r_{1}, \ldots, r_{n}\right)$, if

$$
g_{i}\left(\varepsilon^{r_{1}} x_{1}, \ldots, \varepsilon^{r_{n}} x_{n}\right)=\varepsilon^{r_{i}+p} g_{i}(x) .
$$

A real-valued function, $\Gamma(x)$, is a homogeneous norm on a finite-dimensional vector space $\mathbb{R}^{n}$, if $\Gamma(x)$ is continuous, positive definite, and is homogeneous of degree one with dilation $\left(r_{1}, \ldots, r_{n}\right)$.

\subsection{Appendix 2: finite-time input-to-state stability \\ Consider a system}

$$
\dot{x}=f(x(t), u(t)), f(0,0)=0, x(t) \in \mathbb{R}^{n}, u(t) \in \mathbb{R}^{m},
$$

where $f$ is continuous with respect to $(x(t), u(t))$, and $u(t): \mathbb{R}_{+} \rightarrow \mathbb{R}^{m}$ is measurable and locally essentially bounded. System (62) is locally finite-time input-to-state (FTISS) with respect to $u(t)$ if there exist some neighborhoods $\mathcal{W}_{x} \subset \mathbb{R}^{n}$ of 0 and $\mathcal{W}_{u} \subset \mathbb{R}^{m}$, such that, for initial state $x(0)=x_{0} \in \mathcal{W}_{x}$ and $u(t) \in \mathcal{W}_{u}$, the solution $x(t)$ is defined for $t>0$ and satisfies

$$
\|x\|_{2} \leq \max \left\{\chi\left(\left\|x_{0}\right\|_{2}, t\right), v\left(\|u(t)\|_{\infty}\right)\right\},
$$

where $\chi$ is a generalized $\mathcal{K} \mathcal{L}$-function, and $v$ is a $\mathcal{K}$-function $([19])$.

Consider a continuous, positive definite, and radially unbounded function $V(x)$ on a domain, $E \subset \mathbb{R}^{n}$, that contains the origin; $V(x)$ is a FTISS-Lyapunov function if each solution $x(t)$ and input $u(t)$ satisfy

$$
\begin{aligned}
& \|x(t)\|_{2} \geq \phi_{1}\left(\|u(t)\|_{\infty}\right) \\
\Longrightarrow & \left.\dot{V}(x)\right|_{(1)} \leq-\phi_{2}\left(\|x(t)\|_{2}\right), \forall t \geq 0,
\end{aligned}
$$

where $\phi_{1}$ and $\phi_{2}$ are $\mathcal{K}$-functions, and $\phi_{2}\left(\|x\|_{2}\right) \backsim V(x)^{\alpha}$ for some positive constant $\alpha<1$ ([19]). 